\title{
Exome sequencing revealed a splice site variant in the IQCE gene underlying post-axial polydactyly type A restricted to lower limb
}

\author{
Muhammad Umair ${ }^{1,2}$, Khadim Shah ${ }^{1}$, Bader Alhaddad ${ }^{2,3}$, Tobias B Haack ${ }^{2,3}$, Elisabeth Graf ${ }^{2,3}$, \\ Tim M Strom ${ }^{2,3}$, Thomas Meitinger ${ }^{2}$ and Wasim Ahmad ${ }^{\star, 1}$
}

Polydactyly is characterized by an extra supernumerary digit/toe with or without bony element. To date variants in four genes GLI3, ZNF141, MIPOL1 and PITX1 have been implicated in developing non-syndromic form of polydactyly. The present study involved characterization of large consanguineous family of Pakistani origin segregating post-axial polydactyly type A, restricted to lower limb, in autosomal recessive pattern. DNA of two affected members in the family was subjected to exome sequencing. Sanger sequencing was then followed to validate segregation of the variants in the family members. A homozygous splice acceptor site variant (c.395-1G $>$ A) was identified in the IQCE gene, which completely co-segregated with post-axial polydactyly phenotype within the family. The homozygous variant was absent in different public variant databases, 7000 in-house exomes, 130 exomes from unrelated Pakistani individuals and 215 ethnically matched controls. Mini-gene splicing assay was used to test effect of the variant on function of the gene. The assay revealed loss of first nucleotide of exon 6 , producing a -1 frameshift and a premature stop codon 22 bases downstream of the variant (p.Gly132Valfs*22). The study provided the first evidence of involvement of the IQCE gene in limbs development in humans.

European Journal of Human Genetics (2017) 25, 960-965; doi:10.1038/ejhg.2017.83; published online 10 May 2017

\section{INTRODUCTION}

Polydactyly (Poly = many; dactylos = digits $)$ is mainly characterized by supernumerary digits/toe, which is either fully developed with the bony element or an additive soft tissue without any bone element. Polydactyly is an inherited limb anomaly with an incidence between 5-19/10 000 live births and rudimentary skin tags are the most common ones. ${ }^{1-3}$ Involvement of the lower limbs have been reported to be less common than the upper limbs, left hand less common than the right hand and the right foot is less common than the left foot. ${ }^{4}$ It appears in combination with other phenotypes or a part of some developmental syndrome (syndromic polydactyly) or can appear as an isolated entity without any other malformation (non-syndromic). ${ }^{5}$

According to the most recent classification, non-syndromic polydactyly has been classified into post-axial polydactyly (PAP), axial (central) polydactyly and pre-axial polydactyly (PPD) according to the position of extra digit (s). ${ }^{3,6}$ The PAP involves extra digit at fifth digit/ toe and the least common type, axial (central) polydactyly characterized by duplication of central three digits (second, third or the fourth) and PPD involves a supernumerary digit/toe affecting the first digits/ toe (thumb) ${ }^{7,8}$ In addition, other complex types of polydactylies like palmar/ventral and dorsal type polydactyly, haas-type polysyndactyly and mirror image polydactyly (MIP) have been reported in the literature. $^{3,9}$

The PAP is classified into two broad categories type A and B. It depends on either the development of an extra digit (PAP-type A) or not fully developed (rudimentary) classified as PAP-type B. ${ }^{7}$ PAP-type A is further classified into six genetic types: PAP-type A1, PAP-type A2, PAP-type A3, PAP-type A4, PAP-type A5 and PAP-type A6. ${ }^{6}$ In humans ten loci and four disease causing genes have been reported to cause different types of non-syndromic polydactylies. This includes a GLI family zinc finger 3 gene (GLI3, MIM 165240); a zinc finger protein 141 gene (ZNF141, MIM 194648); a mirror image polydactyly gene (MIPOL1, MIM 606850) and a paired like homeodomain 1 gene (PITX1, MIM 602149) (Table 1).

Previously, we have reported the first locus for autosomal recessive PAP-type A5 and a novel gene ZNF141 in a family segregating PAPtype $6 .{ }^{10,11}$ In the present study, we have presented another consanguineous family of Pakistani origin segregating PAP. Wholeexome sequencing (WES) identified a biallelic splice acceptor site variant (c.395-1G > A; p.Gly132Valfs ${ }^{\star} 22$ ) in the IQCE gene located on the chromosome 7 p22.3.

\section{MATERIALS AND METHODS \\ Study approval \\ The present study was performed following the declaration of Helsinki protocols, approved by the Institutional Review Board (IRB) of Quaid-i- Azam University, Islamabad, Pakistan and Institutional Review Board of Technical University Munich Germany. Written informed consent for con- ducting the study and for publication of radiographs and photographs were obtained from the family members.}

${ }^{1}$ Department of Biochemistry, Faculty of Biological Sciences, Quaid-i-Azam University, Islamabad, Pakistan; ${ }^{2}$ Institute of Human Genetics, Technische Universitat Munchen, Munchen, Germany; ${ }^{3}$ Institute of Human Genetics, Helmholtz Zentrum Munchen, Neuherberg, Germany

${ }^{*}$ Correspondence: Professor W Ahmad, Department of Biochemistry, Faculty of Biological Sciences, Quaid-i-Azam University, Islamabad, Pakistan. Tel/Fax: +92 51 90643003; E-mail: wahmad@qau.edu.pk

Received 3 December 2016; revised 10 April 2017; accepted 13 April 2017; published online 10 May 2017 
Table 1 Characterization of disease causing non-syndromic polydactyly types

\begin{tabular}{|c|c|c|c|c|}
\hline Genes & Disease & Inheritance & Locus & OMIM \\
\hline$U$ & PPD1 & $A D$ & $U$ & 174400 \\
\hline ZRS/SHH & PPD2 & $A D$ & $7 q 36$ & 174500 \\
\hline$U$ & PPD3 & $A D$ & U & 174600 \\
\hline GLI3 & PPD IV & $A D$ & $7 p 14.1$ & 174700 \\
\hline$U$ & HALLUX TYPE & $U$ & 2q31.1-31.2 & 601759 \\
\hline GLI3 & PAPA1 & $A D$ & $7 p 14.1$ & 174200 \\
\hline$U$ & PAPA2 & $A D$ & 13q21-q32 & 602085 \\
\hline$U$ & PAPA3 & $A D$ & $\begin{array}{l}\text { 19p13.1- } \\
\text { p13.2 }\end{array}$ & 607324 \\
\hline$U$ & PAPA4 & $A D$ & $7 q 21-q 34$ & 608562 \\
\hline$U$ & PAPA5 & AR & $\begin{array}{l}13 q 13.3- \\
q 21.2\end{array}$ & 263450 \\
\hline ZNF141 & PAPA6 & AR & $4 p 16.3$ & 615226 \\
\hline MIPOL1 & COMPLEX TYPE; MIP & $A D$ & $14.11 .2-13$ & 606850 \\
\hline PITX1 & $\begin{array}{l}\text { MIRROR IMAGE } \\
\text { POLYDACTYLY }\end{array}$ & $A D$ & $5 q 31.1$ & 602149 \\
\hline ZRS/SHH & HAAS TYPE & $A D$ & $7 q 36$ & 186200 \\
\hline
\end{tabular}

Abbreviations: $A R$, autosomal recessive; $A D$, autosomal dominant; $U$, unknown.

\section{Isolation of genomic DNA}

Peripheral blood samples were obtained from nine individuals including five unaffected (III-2, III-3, III-4, IV-3, IV-7) and four affected (IV-1, IV-2, IV-5, IV-6) in EDTA containing vacutainer sets (BD, Franklin Lakes, NJ, USA). Genomic DNA was extracted from whole blood using GenElute Blood Genomic DNA Kit (Sigma-Aldrich, St Louis, MO, USA). Extracted DNA was quantified using Nanodrop-1000 spectrophotometer (Thermal Scientific, Wilmington, St Louis, MA, USA).

\section{Whole-exome sequencing}

WES was performed using DNA extracted from blood of two affected members (Figure 1a). Enrichment of exomic sequences was carried out using SureSelect XT Human All Exon $50 \mathrm{Mb}$ kit version 5 (Agilent Technologies, Santa Clara, CA, USA). Sequencing was performed on HiSeq 2500 systems (Illumina, San Diego, CA, USA) using Burrows-Wheeler Aligner (http://bio-bwa.sourceforge. net), and all reads were aligned against human assembly hg19 (GRCh37). SAM tools (http://samtools.sourceforge.net/) and PINDEL (http://gmt.genome.wustl. edu/packages/pindel/) were used for variant calling. Subsequently, filtering of the variants was performed with the help of the SAM tools varFilter and custom scripts. All the variants obtained after filtering were then inserted into an inhouse database. Since the pedigree clearly depicts autosomal recessive inheritance of the phenotype (Figure 1a), therefore to discover putative pathogenic (affects function) homozygous recessive variants, we queried the database to show only those variants in both the affected individuals that were rare homozygous and disease causing.

\section{Bioinformatics analysis}

Frequency of the selected variants was further cross checked with in-house 7000 exomes, Exome Variant Server (http://evs.gs.washington.edu/EVS/), Exome Aggregation Consortium ExAC (http://exac.broadinstitute.org/), 1000 Genomes (http://www.1000 genomes.org) and dbSNP (http://www.ncbi.nlm.nih.gov/ $\mathrm{SNP} /$ ). Variants effect on the nearest splice site was predicted using NNSplice (Berkeley, CA, USA), ${ }^{12}$ MutPred Splice (v1.3.2), ${ }^{13}$ SKIPPY ${ }^{14}$ and Human Splice Finder (v2.4.1). ${ }^{15}$

\section{Sanger sequencing validation}

The homozygous sequence variant, identified here, was verified by bidirectional Sanger sequencing in all members of the family. Primers were designed using Exon Primer (http://ihg.gsf.de).

\section{Splicing assay}

To study effect of the variant on splicing mechanism, we employed a mini-gene assay, which was based on a strategy originally developed for exon trapping. ${ }^{16}$ A $\sim 1900$ bp fragment, containing IQCE exon 5-6 and $\sim 350$ bp of the neighboring introns, were PCR-amplified from genomic DNA of affected and normal individual using High Fidelity Phusion polymerase (Thermo Scientific, Pittsburgh, USA). As high fidelity Phusion polymerase produces blunt ended PCR product, therefore 0.7-1 unit of Taq DNA polymerase was added per tube, incubated at $72{ }^{\circ} \mathrm{C}$ for $10 \mathrm{~min}$ and placed on ice. Two distinct primers were designed (forward: 5'-AACAAAACCTCCCATTGTGTGTGCTTGT-3' and reverse: $5^{\prime}$-TGGTTGTAACAAGTGGTTCTGCCCG-3') and the resulting product was cloned into PCDNA (3.1a) TOPO vector using the TOPO TA Cloning according to manufacturer's protocol (Life Technologies, Carlsbad, CA, USA). Plasmids were analyzed by direct Sanger sequencing and then transfected into HeLa cells (105 cells in $3.5 \mathrm{~cm}$ wells). Total RNA was extracted using the Nucleospin RNA extraction kit (Macherey-Nagel Inc, Bethlehem, PA, USA), retrotranscribed with the Superscript III reverse transcriptase (Invitrogen, Carlsbad, CA, USA), and the resulting CDNA was PCR-amplified using primers designed from within exon 5 (forward: 5'AGTCTGACCCAGGCCCT3') and exon 6 (reverse: 5'CTTCTTTAACTCAATAATCTCGTCA3') of the IQCE gene. The amplified products were analyzed on $1.5 \%$ agarose gel electrophoresis and subsequently sequenced by dideoxy chain termination method. All the variants (Supplementary Table 4) have been submitted to the Leiden Open Variation Database (LOVD) at www.LOVD.nl/KREMEN1 (submission ID 00095221; Variant ID 0000154206).

\section{RESULTS}

\section{Clinical description}

Four affected members, in the family presented here, were diagnosed with PAP on the basis of the observed phenotypes and radiological examinations. Although, phenotypic variability was observed among affected individuals (IV-1, IV-2, IV-5, IV-6), but the condition was found restricted only to feet. An affected individual (IV-5) showed brachymetatarsia of 5th toe in the right foot (Figure 1c), and affected individual (IV-6) had PAP only in the right foot (Figure 1d).

Radiological examination of affected individuals (IV-1, IV-2) showed radial varus deviation of 4 th and 5 th toes in both feet, and varus deviation of 5th toe of right foot in affected individual (IV-5). Bilateral syndactyly of 2nd and 3rd toe was observed only in an affected individual (IV-1). All the affected individuals had well developed nails in the extra toes. Radiographical analysis revealed that both 5th and 6th toes (digits) in the affected individuals were attached to the two headed thick broad 5th metatarsal (Figures 1b-d). The 5th metatarsal in the right foot in affected individual (IV-5) had valgus deviation, while both 5th and 6th toes had cubitus varus deviation as well.

All the affected individuals had normal height and weight. Abnormalities involving heart, kidney, nails, teeth, hearing, and brain were not observed in any affected member. Heterozygous carrier individuals had no abnormality, and were phenotypically and clinically indistinguishable from other normal individuals.

\section{WES and sanger sequencing}

To search for disease causing variants, WES was performed at Institute of Human Genetics, Helmholtz Zentrum, Munchen, Germany as described previously. ${ }^{17}$ Variants screening was performed on the basis of autosomal recessive pattern of inheritance of the polydactyly phenotype, rare variants and OMIM list of syndromic and nonsyndromic polydactyly genes presented in Supplementary Table 1. We focused only on pathogenic (affects function), disease causing nonsynonymous (NS) variants, causing splice acceptor and donor site variants (SS), nonsense, missense, short frameshift coding deletions (indel) or insertions and large deletions/duplications. Total 82 
a
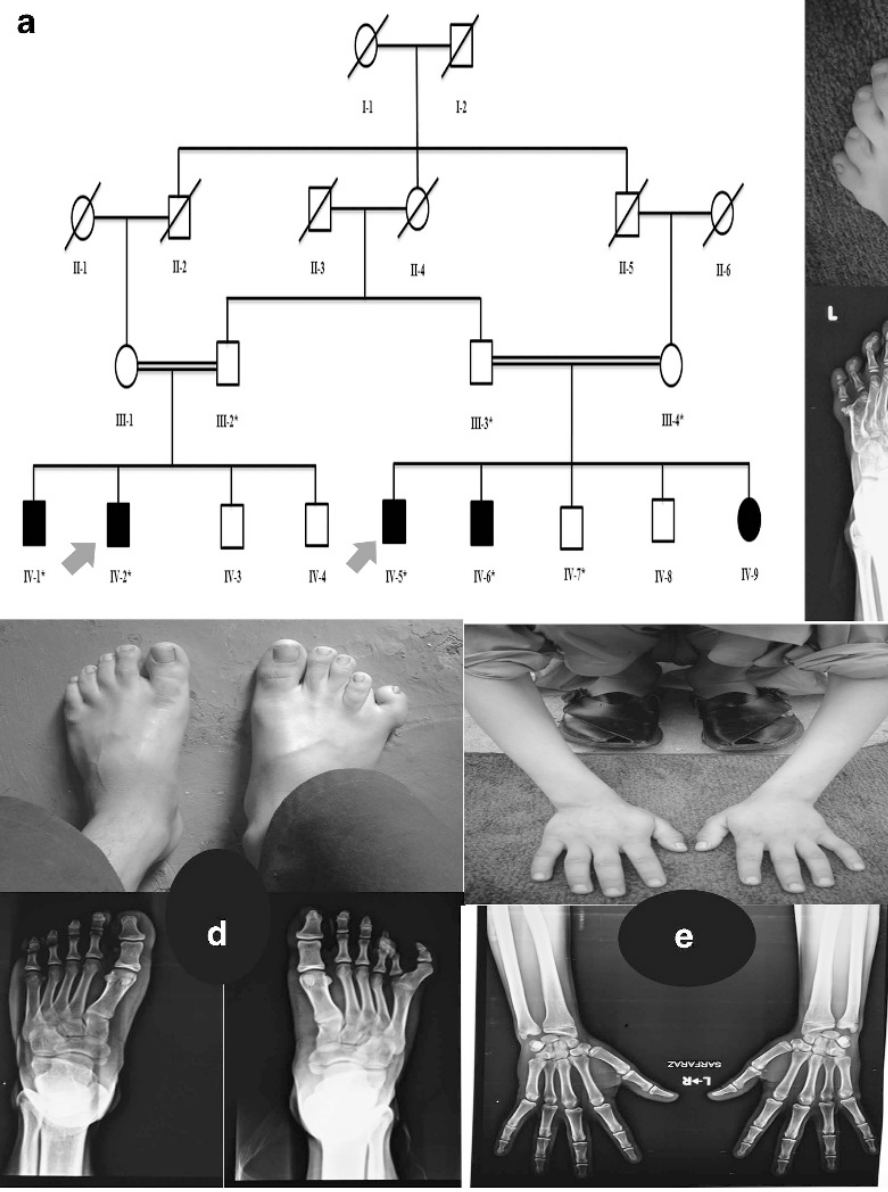
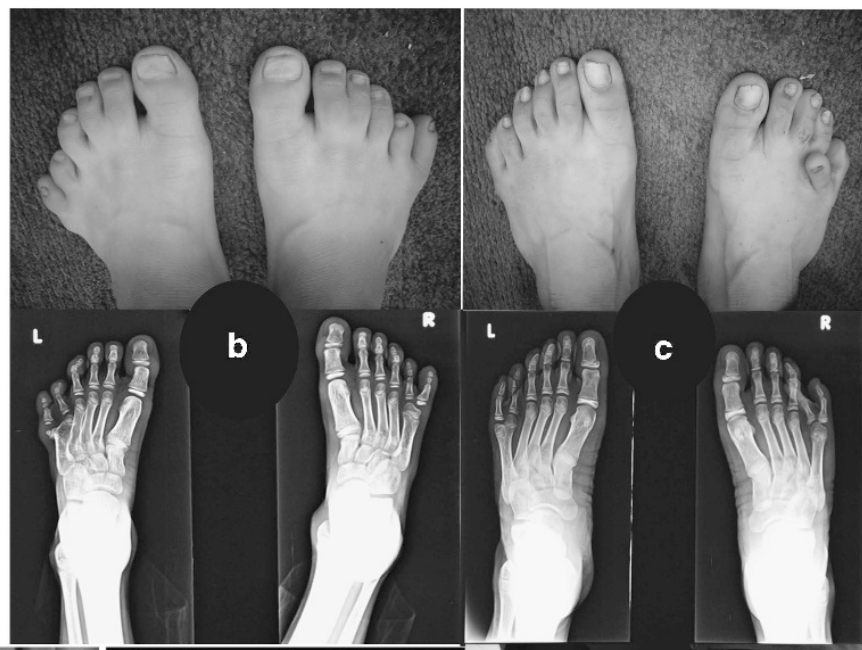

c

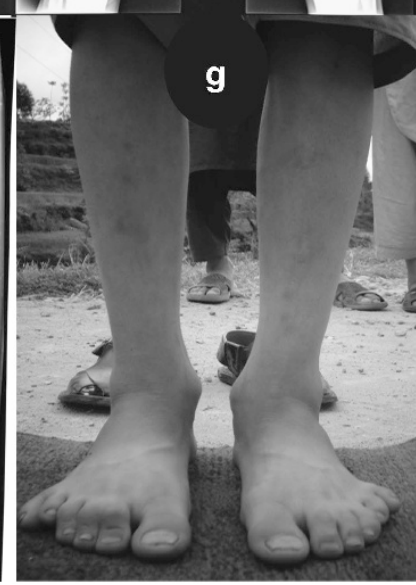

Figure 1 (a) Pedigree depicting autosomal recessive inheritance pattern of PAP. Clear circles and squares represent normal females and males, respectively. Filled symbols representing affected members. Double lines indicate consanguineous unions. Individual numbers labeled with asterisks indicate those who were available for the present study. Red arrows indicating affected individuals subjected to WES. (b) Image and radiographs of an affected individual IV-1 having bilateral PAP and incomplete syndactyly of 2 nd and 3rd toe. Radiographs showing radial varus deviation of 4th and 5th toes in both feet, extra toes originating form 5th metatarsal. (c) Image and radiographs of affected individual (IV-5) having bilateral PAP with brachymetatarsia of 5th toe in the right foot. (d) Image and radiographs of an affected individual IV-6 showing unilateral PAP in the right foot. Radiographic analysis revealed varus deviation of the 5th and radial valgus deviation of the 6th toe with extra toe attached to the 5th metatarsal. (e) Image and radiographs of both hands of an affected individual IV-1 showing absence of polydactyly phenotype. (f) Lower limbs radiographs of IV-1 having normal tibia and fibula. (g) Lower limbs of IV-1 having normal length and normal tibia/fibula. All the affected individuals showed complete well developed nails in the extra toes. The full colour version of this figure is available at European Journal of Human Genetics online.

compound heterozygous or homozygous variants were detected in DNA of at least one of the affected individual subjected for exome sequencing (Supplementary Table 2). Exome statistics are presented in Supplementary Table 3. Considering polydactyly is a rare disorder and it is highly unlikely that the disease causing variant could be present in the general population therefore the data was cross-matched with online public databases.

Following step-by-step filtering process for screening homozygous and compound heterozygous variants, six homozygous variants were identified/filtered in six different genes including MAD1L1 (MIM 602686), IQCE, TNRC18, PNPLA4, LANCL2 and BAG3 (MIM 603883). A splice acceptor site variant c.395-1G > A (NM_152558.4; NC_000007.14), in intron 5 of the IQCE gene, located on chromosome $7 \mathrm{p} 22.3$, was found to segregate with the disease phenotype as verified by Sanger sequencing (Supplementary Figure). The variant (c.395-1G > A) was not found in homozygous state in dbSNP, 1000 Genomes, EVS, internal database and ExAC and predicted to be deleterious in online available bioinformatics tools (Supplementary Table 4). In the ExAc database containing more than 60000 human exome data, the IQCE variant (c.395-1G>A) was found three times in heterozygous state only with allele frequency of 0.0001818 in south Asian population. The variant is highly conserved across multiple species (Figure 2).To exclude polymorphic nature of the variant, it was cross checked within different online variant databases, 7000 in-house exome (IHG, Helmholtz, Munich, Germany), 130 exomes from unrelated Pakistani individuals, and 215 ethnically matched controls. In silico analyses clearly validated that the splice site variant has a strong impact on the normal splicing pattern of the gene, as expected for DNA changes involving -1 and -2 bases of intron acceptor sequences.

HeLa cells transfection using mini-gene constructs bearing this change and its wild-type counterpart revealed that (c.395-1G > A) affects the canonical splicing of exon 6 by knocking down its natural $3^{\prime}$ acceptor splice site and eliciting the use of a cryptic splice site, just one 
a

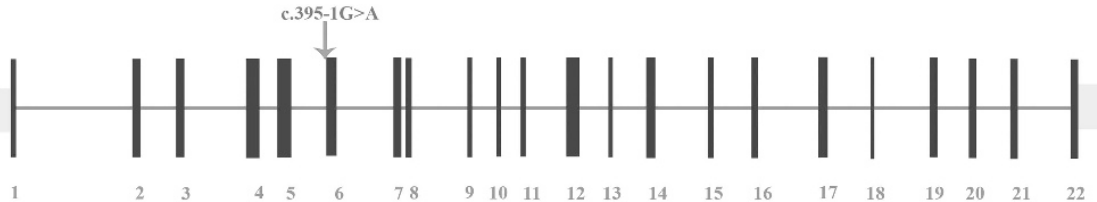

Exon 1

b

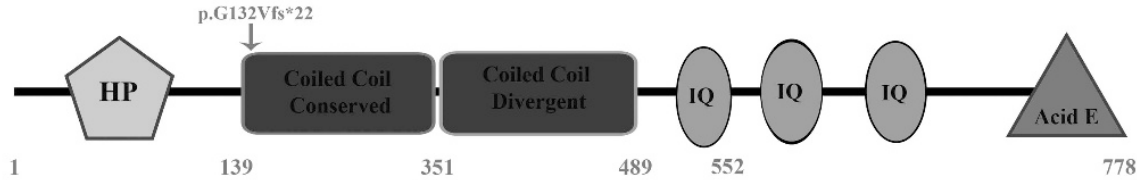

C

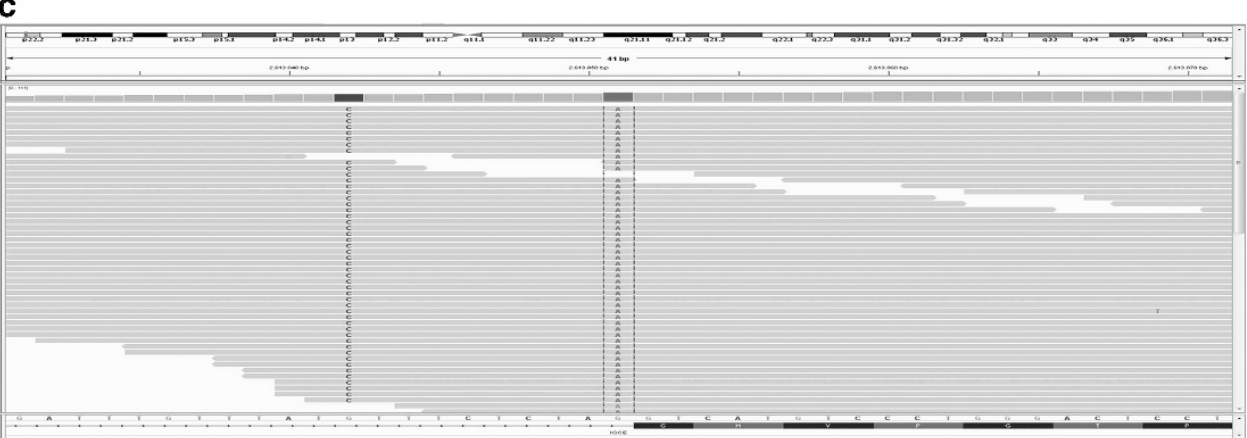

d

* Mutated, Homo sapiens, 153 aa (Mutated):

2. ENSPTRPOOO00032212, Pan troglodytes, 695 aa:

3. ENSNLEP00000013097, Nomascus leucogenys, 696 aa:

4. ENSPPYPO0000019407, Pongo abelii, 684 aa:

5. ENSGGOPOO000020212, Gorilla gorilla, 702 aa:

6. ENSMMUP00000035531, Macaca mulatta, 695 aa:

7. ENSCJAP00000034910, Callithrix jacchus, 698 aa:

8. ENSTBEPOOOOOO1087

9. ENSOGAPOO000017800, Otolemur garnettii, 697 ac

10. ENSMPUP00000017834, Mustela putorius, 782 aa:

11. ENSSSCP00000008083, Sus scrofa, 768 aa:

12. ENSMUSPOO000045913, Mus musculus, 778 aa:

13. ENSECAPOO000019234, Equus caballus, 679 aa:

14. ENSCAF POOOOOO32074, Canis lupus, 754 aa:

15. ENSSTOP00000004143, Ictidomys tridecemlineatus, 697 aa:

16. ENSBTAP00000019288, Bos taurus, 694 aa:

17. ENSTTRPOO000009163, Tursiops truncatus, 625 aa:

18. ENSRNOPOOOOOO40350, Rattus norvegicus, 782 aa:

19. ENSAMEPOOO00003407, Ailuropoda melanoleuca, 534 aa:

20. ENSPVAPO0000007459, Pteropus vampyrus, 616 aa:

20. ENSPVAP00000007459, Pteropus vampyrus, 616 aa:
21. ENSDORPOOO00000772, Dipodomys ordii, 642 aa:

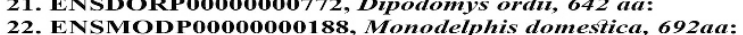

22. ENSWGAIP00000006891, Gallus gallus, 703 aa:

23. ENSGALPO0000006891, Gallus gallus, 703 aa:
24. ENSOANP00000006854, Ornithorhynchus anatinus, 735 aa:

25. ENSDARPOOOOO111024, Danio rerio, 680 aa:

26. ENSGACPOO000021812, Gasterosteus aculeatus, 666 aa:

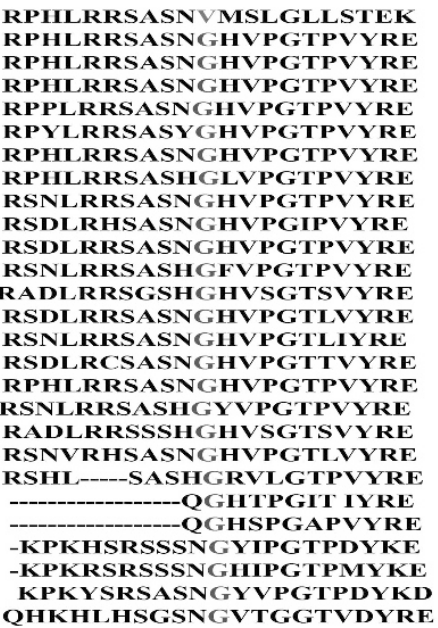

Figure 2 (a) IQCE gene structure showing the identified variant (red arrow). (b) The structure of the gene product (IQCE protein cartoon diagram) showing different domains and location of variant is represented with red arrow. (c) Visualization of WES data under the expert Integrative Genomics Viewer (IGV). The forward strand displaying the novel splice acceptor site variant in intron 5 of IQCE is shown in the affected individual. (d) Conservation of Glycine (G) residue across different species, which is changed into Valine (V) due to first G nucleotide deletion in exon 6 as a result of splice acceptor site variant (c.395$1 \mathrm{G}>\mathrm{A}$ ). Intronic regions are not drawn up to scale. The full colour version of this figure is available at European Journal of Human Genetics online.

base pair downstream of intron 3's acceptor sequence (Figures 3a and $b$ ). This event led to the loss of first nucleotide of exon 6 , producing a -1 frameshift and a premature stop codon 22 bases downstream of the variant (p.Gly132Valfs ${ }^{\star} 22$ ). We were unable to perform protein quantification due to refusal by the affected members to provide additional blood samples and skin biopsies.

\section{DISCUSSION}

In the present study, we have characterized a large consanguineous family segregating post-axial polydactyly type A (PAP-type A) in autosomal recessive manner. PAP phenotypes observed in affected members of the family were similar to those reported previously in families inheriting PAP-type $\mathrm{A}^{11,18-20}$. However, polydactyly restricted to feet only were not reported in these cases. Cutaneous syndactyly, involving 2 nd and 3 rd toes, was observed only in one affected member (IV-1) of the present family. This feature of cutaneous syndactyly, associated with PAP-type A/B in hands/feet, was reported previously by Galjaard et al. ${ }^{19}$ Hallux valgus deformity, reported previously by Kalsoom et al. ${ }^{11}$ was also observed in an affected member of the present family. Fork shaped metatarsal of the 5 th toe, reported by Kalsoom et al., ${ }^{11}$ was missing in affected members of the present study. Instead the 5th and 6th toe were attached to the two headed broad 5th metatarsal. A condition called brachymetatarsia of 5 th toe, observed in an affected individual (IV-6) in the present study, was not reported earlier. 

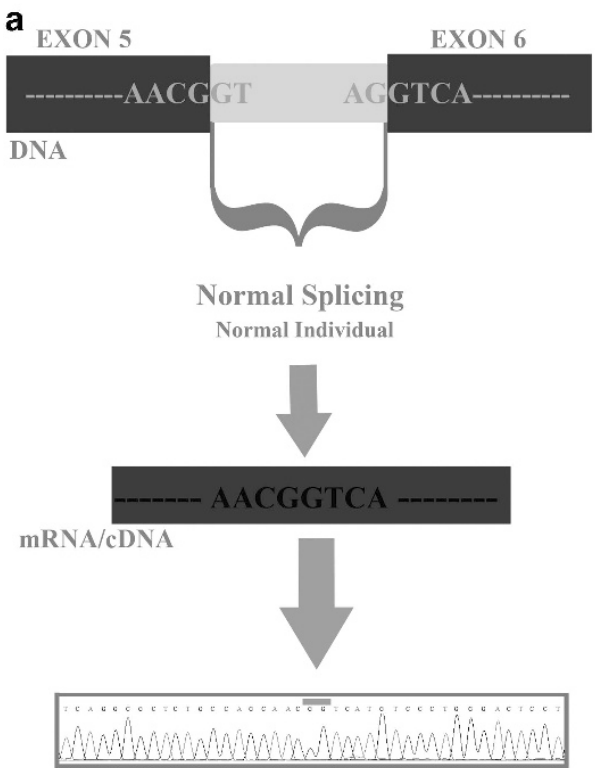

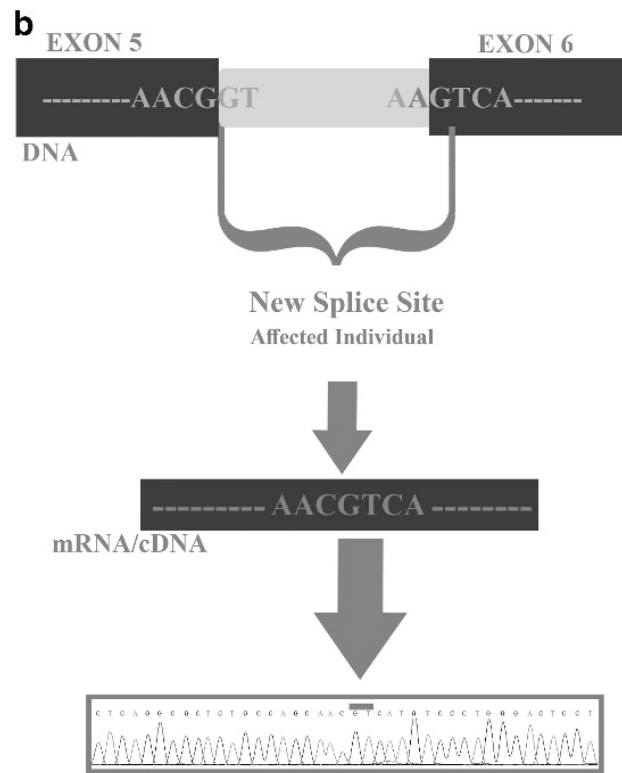

Figure 3 (a) Schematic demonstration of the effect of the splice acceptor site variant (c.395-1G>A) on IQCE mRNA using mini-gene assay technique. cDNA sequence showing construct from control DNA. (b) cDNA sequence showing DNA constructs bearing the variant.

Exome sequencing revealed a homozygous novel splice acceptor site variant (c.395-1G>A; NM_152558.4), segregating with the disease phenotype, in the family reported here. The variant was present in intron 5 of the IQCE gene mapped on chromosome 7p22.3. In silico analysis, verified by in vitro mini-gene assay, revealed the variant (c.395-1G > A) altered the splicing pattern of the IQCE gene, induced use of cryptic splice site and finally produced a frameshift leading to a premature termination codon (p.Gly132Valfs ${ }^{\star 22}$ ). However, since the mini-gene assay only included exons 5 and 6 , therefore this cannot be validated that this is the only splicing alteration produced by the variant in patient cells. The variant, is predicted to result in truncated IQCE protein lacking the coiled coil conserved/diverged regions, three tandem $\alpha$-helical IQ motifs involved in interaction of IQCE with EFCAB7, and C-terminal Acid E domain, affecting protein interactions and most likely degraded by nonsense mediated mRNA decay (NMD). ${ }^{21}$

In the Iqce knockout mouse (MGI:1921489), various types of skeletal deformities including pre-axial polydactyly (1/10 mice showed pre-axial polydactyly only in one lower limb), digit abnormalities, and short and long tibia were reported only in the lower limbs (http://www.mousephenotype.org/data/imageComparator?\&parameter_stable_id = IMPC_XRY_034_001\&acc = MGI:1921489). Additional features observed in Iqce knockout mouse including metabolism, behavior, skeleton, immune system, eyes and cardiovascular system abnormalities, that were missing in our family. ${ }^{22,23}$ In the knockout mice, the gene Iqce was completely removed leading to different phenotypes than those reported in human here. In addition, several other factors including variable expressivity, incomplete penetrance, difference in genetic background, environmental influence and epigenetic phenomena probably played roles in developing differential phenotypes in human. Presence of long/short tibia phenotype in the knockout mice supported the notion that Iqce pathogenesis causes defects in lower limbs, thus supporting the data presented here.

EVC and EVC2 form a protein complex which has been identified as a tissue specific regulator of $\mathrm{Hh}$ signaling. ${ }^{24-26} \mathrm{EVC}$ and EVC2 bind to SMO and localize to EVC zone at the base of primary cilia, which is critical for Hh signaling. The IQCE-EFCAB7 module acts tethering the EVC-EVC2 complex (the EvC complex) to the base of the primary cilium. ${ }^{21}$ EFCAB7 and IQCE both co-localize with EVC2 at the base of cilia in the EVC zone. Biochemical interactions and subcellular localization of IQCE and EFCAB7 suggested that they have important role in EVC-EVC2 complex regulation and regulating Hh signaling transduction. ${ }^{21,27}$

Dorn et al. ${ }^{28}$ suggested that EVC-EVC2 complex is involved in regulation of SMO-GLI step in Hh signaling. Pusapati et al..$^{21}$ verified experimentally that IQCE-EFCAB7 complex play an important role in the step between SMO-GLI proteins and depletion of both IQCE and EFCAB7 affect the signaling by SMO and in turn affect Hh signaling.

In conclusion, we have used WES to identify the first sequence variant in the IQCE gene resulting in a non-syndromic form of PAP. This provided the first evidence in human that missing IQCE lead to abnormal limb development.

\section{CONFLICT OF INTEREST}

The authors declare no conflict of interest.

\section{ACKNOWLEDGEMENTS}

We highly appreciate cooperation and participation of the family members in this study. Muhammad Umair and Khadim Shah were supported by International Research Support Initiative Program (IRSIP) and Indigenous PhD fellowship (HEC) and from Higher Education Commission (HEC), Islamabad, Pakistan. This work was funded by Pakistan Academy of Science (PAS) Islamabad, Pakistan to Wasim Ahamad.

\footnotetext{
1 Schwabe GC, Mundlos S: Genetics of congenital hand anomalies. Handchir Mikrochir Plast Chir 2004; 36: 85-97.

2 Christensen JC, Leff FB, Lepow GM et al: Congenital polydactyly and polymetatarsalia: classification, genetics, and surgical correction. J Foot Ankle Surg 2011; 50: 336-339.

3 Malik S: Polydactyly: phenotypes, genetics and classification. Clin Genet 2014; 85: 203-212.

4 Malik S, Ullah S, Afzal M, Lal K, Haque S: Clinical and descriptive genetic study of polydactyly: a Pakistani experience of 313 cases. Clin Genet 2014; 85: 482-486.
} 
5 Biesecker LG: Polydactyly: how many disorders and how many genes? 2010 update. Develop Dyn 2011; 240: 931-942.

6 Deng H, Tan T, Yuan L: Advances in the molecular genetics of non-syndromic polydactyly. Expert Rev Mol Med 2015; 17: 1-10.

7 Talamillo A, Bastida MF, Fernandez-Teran M, Ros MA: The developing limb and the control of the number of digits. Clin Genet 2005; 67: 143-153.

8 Haber LL, Adams HB, Thompson GH, Duncan LS, Didomenico LA, McCluskey WP: Unique case of polydactyly and a new classification system. J Pediatr Orthop 2007; 27: 326-328.

9 Phadke SR, Sankar VH: Polydactyly and genes. Indian J Pediatr 2010; 77: 277-281.

10 Umm-e-Kalsoom, Basit S, Kamran-ul-Hassan Naqvi S, Ansar M, Ahmad W: Genetic mapping of an autosomal recessive postaxial polydactyly type A to chromosome 13q13.3q21.2 and screening of the candidate genes. Hum Genet 2012; 131: 415-422.

11 Kalsoom UE, Klopocki E, Wasif $\mathrm{N}$ et al: Whole exome sequencing identified a nove zinc-finger gene ZNF141 associated with autosomal recessive postaxial polydactyly type A. J Med Genet 2012; 50: 47-53.

12 Reese MG, Eeckman FH, Kulp D, Haussler D: Improved splice site detection in Genie. J Comput Biol 1997; 4: 311-323.

13 Mort M, Sterne-Weiler T, Li B et al: MutPred Splice: machine learning-based prediction of exonic variants that disrupt splicing. Genome Biol 2014; 15: R19.

14 Woolfe A, Mullikin JC, Elnitski L: Genomic features defining exonic variants that modulate splicing. Genome Biol 2010; 11: R20.

15 Desmet FO, Hamroun D, Lalande M, Collod-Béroud G, Claustres M, Béroud C: Human Splicing Finder: an online bioinformatics tool to predict splicing signals. Nucleic Acids Res 2009; 37: e67.

16 Buckler AJ, Chang DD, Graw SL: Exon amplification: a strategy to isolate mammalian genes based on RNA splicing. Proc Natl Acad Sci USA 1991; 88: 4005-4009.

17 Haack TB, Danhauser K, Haberberger B et al: Exome sequencing identifies ACAD9 mutations as a cause of complex I deficiency. Nat Genet 2010; 42: 1131e4.
18 Zhao H, Tian Y, Breedveld G et al: Postaxial polydactyly type A/B (PAP-A/B) is linked to chromosome 19p13.1-13.2 in a Chinese kindred. Eur J Hum Genet 2002; 10: 162-166.

19 Galjaard RJ, van der Linde $\mathrm{HC}$, Eussen $\mathrm{BH}$ et al: Isolated postaxial polydactyly type B with mosaicism of a submicroscopic unbalanced translocation leading to an extended phenotype in offspring. Am J Med Genet A 2003; 121A: 168-173.

20 Al-Qattan MM: A novel frameshift mutation of the GLI3 gene in a family with broad thumbs with/without big toes, postaxial polydactyly and variable syndactyly of the hands/feet. Clin Genet 2012; 82: 502-504.

21 Pusapati GV, Hughes CE, Dorn KV et al: EFCAB7 and IQCE regulate hedgehog signaling by tethering the EVC-EVC2 complex to the base of primary cilia. Dev Cell 2014; 28 483-496.

22 Hansen GM, Markesich DC, Burnett MB et al: Large-scale gene trapping in C57BL/6 N mouse embryonic stem cells. Genome Res 2008; 18: 1670-1679.

23 Eppig JT, Blake JA, Bult CJ, Kadin JA, Richardson JE: The Mouse Genome Database Group. 2015. The Mouse Genome Database (MGD): facilitating mouse as a model for human biology and disease. Nucleic Acids Res 2015; 28: 43.

24 Blair HJ, Tompson S, Liu YN et al: Evc2 is a positive modulator of Hedgehog signalling that interacts with Evc at the cilia membrane and is also found in the nucleus. BMC Biol 2011; 9: 14.

25 Ruiz-Perez VL, Blair HJ, Rodriguez-Andres ME et al: Evc is a positive mediator of Ihhregulated bone growth that localises at the base of chondrocyte cilia. Development 2007; 134: 2903-2912.

26 Caparrós-Martín JA, Valencia M, Reytor E et al: The ciliary Evc/Evc2 complex interacts with Smo and controls Hedgehog pathway activity in chondrocytes by regulating Sufu/ Gli3 dissociation and Gli3 trafficking in primary cilia. Hum Mol Genet 2013; 22 : 124-139.

27 Lopez-Rios J: The many lives of SHH in limb development and evolution. Semin Cell Dev Biol 2016; 49: 16-24.

28 Dorn KV, Hughes CE, Rohatgi R: A Smoothened-Evc2 complex transduces the Hedgehog signal at primary cilia. Dev Cell 2012; 23: 823-835.

Supplementary Information accompanies this paper on European Journal of Human Genetics website (http://www.nature.com/ejhg) 